\title{
COINTER PDVS 2021 \\ A QUALIDADE DO SONO DOS PROFISSIONAIS DE SAÚDE DURANTE A PANDEMIA: UMA REVISÃO DE LITERATURA
}

\section{LA CALIDAD DEL SUEÑO DE LOS PROFESIONALES DE LA SALUD DURANTE LA PANDEMIA: UNA REVISIÓN DE LA LITERATURA}

\section{THE QUALITY OF SLEEP OF HEALTH PROFESSIONALS DURING PANDEMIC: A LITERATURE REVIEW}

\author{
Apresentação: Comunicação Oral \\ Danielle Pereira de Lima ${ }^{1}$; Eduardo Ramos da Silva ${ }^{2}$; Girlâyne Fragoso Alves da Silva ${ }^{3}$ Juliana Mendes Correia ${ }^{4}$; \\ Viviany Andrea Alves Meireles 5
}

\section{RESUMO}

DOI: https://doi.org/10.31692/2526-7701.IIICOINTERPDVS.0063

O objetivo foi averiguar através de uma revisão de literatura o quão afetado foram os profissionais de saúde com relação ao sono, e se há alguma maneira de tentar amenizar os impactos causados pela pandemia nos profissionais de saúde. A pandemia COVID-19 tem o potencial de afetar a saúde mental das pessoas e principalmente dos profissionais de saúde, que se encontram na linha da frente desta crise. A insônia está frequentemente relacionada com a exposição a situações estressantes, tais como a atual crise de saúde, bem como outras perturbações mentais, condições físicas e problemas relacionados com o trabalho. A pandemia Covid-19 é um reflexo que agrava as crises da sociedade, os sintomas das doenças que existia antes da pandemia como depressão, ansiedade, problemas de sono, se destacam de uma forma gritante, e um desses sintomas é o cansaço. Os contagiados são acometidos de um extremo abatimento e esgotamento; para os curados, uma das sequelas é justamente a síndrome da fadiga, que é muito mais do que um simples cansaço. Os sem sintomas ou considerados saudáveis em home office se cansam mais do que quando trabalham presencialmente, já que é um trabalho que necessita de rituais e de estruturas temporárias fixas. Trata-se de uma revisão de literatura integrativa, que segue a seguinte pergunta norteadora "qual o impacto da pandemia nos profissionais de saúde?". A revisão de literatura foi realizada no período de Junho a Agosto do ano de 2021, utilizando as principais bases de dados disponíveis com grande número de publicação na área da saúde, como a Scientific Electronic Library Online (SciELO), Google Scholar, Medical Literature Analysis and Retrieval System Online (MEDLINE) e Biblioteca Virtual de Saúde (BVS). Os estudos mostraram que a pandemia afetou o dia a dia dos profissionais de saúde, como tristeza, ansiedade, depressão, irritabilidade, falta de apetite. principal fator de risco associado à insônia era trabalhar num ambiente de alto risco, seguido pelo sexo feminino e ter um nível educacional mais baixo. O estado emocional tem um papel importante na qualidade do sono, então, o sono é o momento de recuperação do organismo, com a eliminação de alguns hormônios que permitem a regeneração dos grupos musculares. Além disso, todos precisam atentar-se para a real importância do sono para a saúde humana, entre sono e indicadores de saúde, o mais importante que a duração do sono é a qualidade do sono.

\footnotetext{
${ }^{1}$ Fonoaudiologia, Centro Universitário São Miguel, fgadanielle@ gmail.com

${ }^{2}$ Fonoaudiologia, Centro Universitário São Miguel, eduardo.ramos.fono@gmail.com

${ }^{3}$ Fonoaudiologia, Universidade Católica de Pernambuco, girlayne.fragoso@gmail.com

${ }^{4}$ Doutora, UNISÃOMIGUEL, IIDV-PDVS, mendescoreia@yahoo.com.br

${ }^{5}$ Doutora em Nutrição, UNISÃOMIGUEL e UFPE, vivianymeireles00@gmail.com
} 


\title{
A QUALIDADE DO SONO DOS PROFISSIONAIS DE SAÚDE
}

Palavras-Chave: Covid-19, Higiene do sono, Acesso à Informação de Saúde.

\section{RESUMEN}

El objetivo era averiguar, a través de una revisión bibliográfica, cómo se vieron afectados los profesionales de la salud en relación con el sueño, y si hay alguna forma de intentar mitigar los impactos causados por la pandemia en los profesionales de la salud. La pandemia de COVID-19 puede afectar a la salud mental de las personas y, en especial, de los profesionales sanitarios, que están en primera línea de esta crisis. El insomnio suele estar relacionado con la exposición a situaciones de estrés, como la actual crisis sanitaria, así como con otros trastornos mentales, afecciones físicas y problemas relacionados con el trabajo. La pandemia de Covid-19 es un reflejo que agrava las crisis de la sociedad, los síntomas de las enfermedades que existían antes de la pandemia como la depresión, la ansiedad, los problemas de sueño, resaltan de manera flagrante, y uno de estos síntomas es la fatiga. Los infectados se ven afectados por un desánimo y un agotamiento extremos; para los curados, una de las secuelas es precisamente el síndrome de fatiga, que es mucho más que un simple cansancio. Las personas sin síntomas as consideradas sanas en el home office se cansan más que cuando trabajan presencialmente, ya que es un trabajo que necesita rituales y estructuras temporales fijas. Se trata de una revisión bibliográfica integradora, que sigue la siguiente pregunta orientadora: "¿cuál es el impacto de la pandemia en los profesionales sanitarios?". La revisión de la literatura se realizó en el período de junio a agosto de 2021, utilizando las principales bases de datos disponibles con un gran número de publicaciones en el área de la salud, como Scientific Electronic Library Online (SciELO), Google Scholar, Medical Literature Analysis and Retrieval System Online (MEDLINE) y Virtual Health Library (BVS). Los estudios mostraron que la pandemia afectaba a la vida diaria de los profesionales sanitarios, como la tristeza, la ansiedad, la depresión, la irritabilidad, la falta de apetito. El principal hecho de riesgo asociado al insomnio era trabajar en un entorno de alto riesgo, seguido del sexo femenino y de tener un nivel educativo más bajo. El estado emocional juega un papel importante en la calidad del sueño, por lo que el sueño es el momento para que el cuerpo se recupere, con la eliminación de algunas hormonas que permiten la regeneración de los grupos musculares. Además, todo el mundo debe prestar atención a la importancia real del sueño para la salud humana, entre los indicadores de sueño y salud, lo que es más importante que la duración del sueño es la calidad del mismo.

Palabras Clave: Covid-19, Higiene del sueño, acceso a la información de salud.

\begin{abstract}
The objective was to find out through a literature review how health professionals were affected with regard to sleep, and if there is any way to try to mitigate the impacts caused by the pandemic on health professionals. The COVID-19 pandemic has the potential to affect the mental health of people and especially healthcare professionals, who are on the front lines of this crisis. Insomnia is often related to exposure to stressful situations, such as the current health crisis, as well as other mental disorders, physical conditions, and work-related problems. The Covid-19 pandemic is a reflection that aggravates the crises in society, the symptoms of the diseases that existed before the pandemic such as depression, anxiety, sleep problems, stand out in a glaring way, and one of these symptoms is fatigue. Those who are infected are affected by extreme exhaustion; for those who are cured, one of the consequences is precisely the fatigue syndrome, which is much more than just tiredness. Those without symptoms or considered healthy in home office get tired more than when they work face-to-face, since it is a job that needs rituals and fixed temporary structures. This is an integrative literature review, following the following guiding question "what is the impact of the pandemic on healthcare professionals?" The literature review was conducted from June to August 2021, using the main available databases with a large number of publications in the health area, such as Scientific Electronic Library Online (SciELO), Google Scholar, Medical Literature Analysis and Retrieval System Online (MEDLINE) and Virtual Health Library (VHL). The studies showed that the pandemic affected the health professionals' daily lives, such as sadness, anxiety, depression, irritability, lack of appetite. The main risk factor associated with insomnia was working in a high-risk environment, followed by being female and having a lower educational level. The emotional state plays an important role in the quality of sleep, so sleep is the time for the body to recover, with the elimination of some hormones that allow the regeneration of muscle groups. Moreover, everyone needs to pay attention to the real importance of sleep for human health, between sleep and health indicators, what is more important than the duration of sleep is the quality of
\end{abstract}


sleep.

Keywords: Covid-19, Sleep Hygiene, Access to Health Information.

\section{INTRODUÇÃO}

No Brasil, devido a pandemia, em 9 de agosto de 2020, foi atingido um nível de 100 mil mortes atribuídas à COVID-19. Um estudo realizado na China revelou que os profissionais de saúde que estavam na vanguarda da luta contra a COVID-19 estavam em alto risco de depressão, ansiedade, insônia e sintomas de stress pós-traumático. Por isso a hipótese de que todos os profissionais de saúde tiveram um impacto negativo na qualidade de vida durante a pandemia ao avaliarem juntos a dieta, a atividade física, o consumo de álcool e o sono. Uma hipótese secundária é que os médicos relatariam piores resultados nos hábitos de sono (MOTA, I. A., et al. 2021).

Diante da falta de medidas de controle suficientes e à falta de abordagens terapêuticas eficazes a situação agravou-se, para além das precárias infraestruturas de saúde pública. Estas incertezas tiveram consequências em vários setores na rotina da vida das pessoas, com implicações na saúde mental dos indivíduos, especialmente entre os profissionais de saúde, pois tiveram de lidar com o contexto extremamente adverso na pandemia (BRITO-MARQUES, et al, 2021).

Serrano-Ripoll, M.J., et al (2021) afirmam que a pandemia COVID-19 tem o potencial de afetar a saúde mental das pessoas e principalmente dos profissionais de saúde, que se encontram na linha da frente desta crise. A insônia está frequentemente relacionada com a exposição a situações estressantes, tais como a atual crise de saúde, bem como outras perturbações mentais, condições físicas e problemas relacionados com o trabalho., exemplo disso temos os profissionais de saúde que estão e/ou estiveram na linha de frente na pandemia.

Devido das incertezas causadas pela pandemia, vale salientar que todos estão diretamente ou indiretamente afetados pela pandemia, mesmo que alguns não padeceu de necessidades financeiras, mas tiveram perdas irreparáveis, e a pandemia com certeza deixará sequelas, prejuízos. De acordo com os expostos, o objetivo é averiguar através de uma revisão de literatura o quão afetado foram os profissionais de saúde com relação ao sono, e se há alguma maneira de tentar amenizar os impactos causados pela pandemia nos profissionais de saúde.

\section{FUNDAMENTAÇÃO TEÓRICA}

Em 17 de Março de 2020 o primeiro óbito por COVID-19 foi notificado no Brasil, 20 dias após o registro do primeiro caso, a doença já havia sido declarada como pandêmica pela Organização Mundial da Saúde (OMS). A Europa acumulava mais de 64 mil casos e 3 mil 


\section{A QUALIDADE DO SONO DOS PROFISSIONAIS DE SAÚDE}

mortes, sendo a Itália o país mais afetado (WHO,2020).

Barros e colaboradores (2020), relatam que o contexto pandêmico e as medidas de controle preconizadas afetam a população em muitas dimensões das condições de vida e de saúde e, entre elas, de forma significativa, o componente de saúde mental. A presença de transtornos mentais, sofrimento psíquico e alterações do sono exerce reconhecidos efeitos negativos no cotidiano e na qualidade de saúde e de vida das pessoas, contribuindo com percentual relevante de anos vividos com incapacidades.

$\mathrm{O}$ atual cenário pandêmico não só alterou a rotina de grande parte das pessoas, mas também afetou a qualidade do sono, incluindo daquelas que estão na linha de frente desde o início. Diante disso, muito se tem discutido sobre o sono e sua função imunológica, algo mais evidente na avaliação de indivíduos em privação de sono, quando há aumento da atividade de citocinas, como o interferon (IFN), fator de necrose tumoral alfa (TNF- $\alpha$ ), e interleucina-1-beta (IL-1-beta), além de aumento de marcadores inflamatórios como a proteína $\mathrm{C}$ reativa (PCR), (RUIZ; TUFIK, 2014).

A pandemia Covid-19 é um reflexo que agrava as crises da sociedade, os sintomas das doenças que existia antes da pandemia como depressão, ansiedade, problemas de sono, se destacam de uma forma gritante, e um desses sintomas é o cansaço (BARROS et al., 2020). Os contagiados são acometidos de um extremo abatimento e esgotamento; para os curados, uma das sequelas é justamente a síndrome da fadiga, que é muito mais do que um simples cansaço. Os sem sintomas ou considerados saudáveis em home office se cansam mais do que quando trabalham presencialmente, já que é um trabalho que necessita de rituais e de estruturas temporárias fixas.

Trabalhar sozinho, na frente da tela do computador, e a falta de contatos sociais é exaustiva. Tudo isso gera um impacto, porque todas as pandemias são geradoras de forte impacto social, econômico e político (DIAS, E., 2021). Quando as horas de sono necessárias não são alcançadas seja por algumas etiologias orgânicas, psicológicas, ambientais e/ou sociais desencadeiam se os distúrbios do sono Neves, Macedo e Gomes (2017), que têm sido intensificados ao longo da pandemia da COVID-19, uma vez que a exposição a fatores que estressam é maior (BLUME et al., 2020).

Assim, durante o período de quarentena, foi constatado uma redução no bem-estar mental e físico das pessoas em geral e principalmente os profissionais de saúde, e essa condição esteve relacionada à redução da qualidade do sono (BLUME et al., 2020). Nesse cenário, dentre os transtornos que mais afetam o sono, o mais comum é a insônia, seja do tipo inicial, de manutenção ou terminal, com duração curta ou crônica. Esta é comum não só na população em 
geral, mas também em populações específicas como de estudantes universitários (CESPEDES E SOUZA, 2020).

De acordo com Lima, M.G. e colaboradores (2021), o sono de boa qualidade tem forte relação com as questões emocionais, com a saúde física e com o bem-estar, atuando nos sistemas imune, metabólico, endócrino e nos processos inflamatórios. Autores alertam que o sono ruim pode estar relacionado com a suscetibilidade à infecção por COVID-19, estudos em diferentes países têm encontrado alta prevalência da qualidade do sono ruim durante a pandemia, chegando a taxas de $57 \%$, na Itália.

A associação entre saúde mental e qualidade do sono tem sido consistentemente reportada. Pesquisa desenvolvida em município brasileiro detectou que a presença de transtornos mentais comuns se associava a um aumento de $61 \%$ na prevalência de má qualidade do sono, mesmo após ajuste por variáveis sociodemográficas, comportamentais e de condição de saúde. Uma metanálise com 21 estudos longitudinais, desenvolvidos entre 1980 e 2010, confirmou a insônia como fator de risco para depressão, com razão de chances (odds ratio) de 2,10. Além disso, estudo longitudinal conduzido na China identificou um relacionamento bidirecional entre sono e otimismo, sendo que o humor depressivo intermediava total ou parcialmente a relação (BARROS, 2020).

Os esforços para conter a disseminação da COVID-19 impuseram o distanciamento físico imprevisível e comumente mandatório em vários países, fazendo com que as pessoas evitassem seus grupos de contato social. Essas medidas fundamentais e necessárias, provavelmente terão um efeito substancial, não apenas na economia e na sociedade, mas também na saúde mental, no estilo de vida e no bem-estar dos indivíduos (ORBEN, TOMOVA E BLAKEMORE, 2020).

Com os profissionais de saúde aconteceram justamente ao contrário, não tiveram o distanciamento e sim estar na linha de frente para os cuidados dos pacientes acometidos pela COVID-19. Prado et al (2020) afirmam que outros autores pesquisaram profissionais da saúde de hospitais com altas taxas de COVID-19 e outros com baixas ou sem casos do vírus, demonstrando que os da linha de frente na pandemia apresentavam mais sintomas de doenças mentais e os trabalhadores da saúde também relataram que suas maiores preocupações e que afetavam a saúde mental era a alta taxa de transmissão do vírus entre a população e seu alto risco de mortalidade.

Além do receio do próprio contágio, esses profissionais da saúde temiam a infecção à sua família, colegas de trabalho, os amigos, sentindo incertezas e rotulações, relutâncias em ir trabalhar e altos índices de pedidos de demissão. Existem relatos de profissionais que diziam 


\section{A QUALIDADE DO SONO DOS PROFISSIONAIS DE SAÚDE}

que sentiram emoções nunca vivenciadas. Esses profissionais estão trabalhando isoladamente, em alta exposição aos riscos e tiveram contato com colegas de trabalho que foram contaminados e alguns foram ao óbito, em um período de aproximadamente quatro meses (KANG, et al., 2020).

Os profissionais de saúde estão sob estresse constante, tem que lidar com doenças, sofrimentos, as mortes e um cronograma irregular de trabalho, com vários plantões o que acaba contribuindo de forma negativa no sono (PHILIPPAR et al., 2021). Ainda de acordo com Jahrami, et al. (2021) existe o fato de tentarem se manter saudáveis, como também seus familiares, tudo isso colaborando com os distúrbios agudos do sono, assim como baixa qualidade de sono e diminuição da duração.

\section{METODOLOGIA}

Trata-se de uma revisão de literatura integrativa, que segue a seguinte pergunta norteadora “qual o impacto da pandemia nos profissionais de saúde?”. A revisão foi realizada no período de Junho a Agosto do ano de 2021, utilizando as principais bases de dados disponíveis com grande número de publicação na área da saúde, como a Scientific Electronic Library Online (SciELO), Google Scholar, Medical Literature Analysis and Retrieval System Online (MEDLINE) e Biblioteca Virtual de Saúde (BVS). Também foi realizado uma busca manual , de um livro publicado no ano de 2014. E outras buscas manuais em jornais on-line.

Na busca foi definido o operador booleano AND para a combinação das palavras chaves determinadas, como insônia AND pandemia, sono AND Covid-19, usando também agrupamento de sinônimos: pandemia covid-19 AND falta de sono, pandemia covid-19 and fadiga. Foram encontrados 2 artigos na MEDLINE, 13 artigos no Google Scolar, 5 artigos na BVS e 10 artigos na SciELO.

Não foram definidos critérios de exclusão para ano ou país de publicação, idioma ou tipo de estudo, visando ampla busca. Entretanto, foi necessário que os achados fossem referentes ao sono da população adulta. Foi realizado a leitura do título e resumo de 30 artigos, sendo excluídos aqueles que não atendiam aos critérios pré-estabelecidos, foram escolhidos 21 artigos que foram lidos na íntegra.

Quadro 01: Títulos dos Artigos foram utilizados nesse trabalho

Título dos Artigos

Ano de publicação 


\begin{tabular}{|c|c|}
\hline $\begin{array}{l}\text { Relato de tristeza/depressão, nervosismo/ansiedade e problemas de } \\
\text { sono na população adulta brasileira durante a pandemia de COVID- } \\
19 .\end{array}$ & 2020 \\
\hline $\begin{array}{c}\text { Impact of COVID-19 pandemic on the sleep quality of medical } \\
\text { professionals in Brazil de produto ou pontual. }\end{array}$ & 2021 \\
\hline $\begin{array}{l}\text { Effects of the COVID-19 lockdown on human sleep and rest-activity } \\
\text { rhythms. }\end{array}$ & 2020 \\
\hline $\begin{array}{l}\text { Hábitos e distúrbios do sono nos estudantes de medicina da } \\
\text { Universidade Estadual de Mato Grosso do Sul. }\end{array}$ & 2020 \\
\hline Educação, a pandemia e a sociedade do cansaço. Ensaio & 2021 \\
\hline Impacto da pandemia da covid-19 na qualidade de vida dos idosos. & 2021 \\
\hline $\begin{array}{l}\text { Associação das condições sociais e econômicas com a incidência dos } \\
\text { problemas com o sono durante a pandemia de COVID-19. }\end{array}$ & 2021 \\
\hline $\begin{array}{l}\text { Impact of COVID-19 on eating habits, physical activity and sleep in } \\
\text { Brazilian healthcare professionals. }\end{array}$ & 2021 \\
\hline $\begin{array}{l}\text { The effects of social deprivation on adolescent developmentand } \\
\text { mental health. }\end{array}$ & 2021 \\
\hline Aspectos imunológicos do sono. & 2014 \\
\hline $\begin{array}{l}\text { Insomnia and sleep quality in healthcare workers fighting against } \\
\text { COVID-19: a systematic review of the literature and meta-analysis. }\end{array}$ & 2021 \\
\hline $\begin{array}{l}\text { WORLD HEALTH ORGANIZATION. WHO Coronavirus Disease } \\
\text { (COVID-2019) situation reports. }\end{array}$ & 2021 \\
\hline $\begin{array}{l}\text { A saúde mental dos médicos em Wuhan, China, lidando com o novo } \\
\text { coronavírus de } 2019 .\end{array}$ & 2020 \\
\hline $\begin{array}{l}\text { A saúde mental dos profissionais frente à pandemia do COVID-19: } \\
\text { uma revisão integrativa. }\end{array}$ & 2020 \\
\hline $\begin{array}{c}\text { Sleep disorders in healthcare professionals in the covid outbreak - 19: } \\
\text { an integrative literature review }\end{array}$ & 2021 \\
\hline $\begin{array}{l}\text { O exame da qualidade do sono para os profissionais de saúde da linha } \\
\text { de frente durante o surto de COVID- } 19 .\end{array}$ & 2021 \\
\hline $\begin{array}{l}\text { The effects of social support on sleep quality of medical staff treating } \\
\text { patients with coronavirus disease } 2019 \text { (COVID-19) in January and } \\
\text { February } 2020 \text { in China. }\end{array}$ & 2020 \\
\hline
\end{tabular}




\section{A QUALIDADE DO SONO DOS PROFISSIONAIS DE SAÚDE}

\begin{tabular}{|c|c|}
\hline $\begin{array}{c}\text { Prevalência de depressão, ansiedade e insônia entre os profissionais de } \\
\text { saúde durante a pandemia COVID-19: Revisão sistemática e meta- } \\
\text { análise. }\end{array}$ & 2020 \\
\hline $\begin{array}{c}\text { The impact of SARS-CoV-2 on the mental health of healthcare } \\
\text { workers in a hospital setting - A Systematic Review. }\end{array}$ & 2020 \\
\hline $\begin{array}{c}\text { Fatores associados aos resultados de saúde mental entre os } \\
\text { profissionais de saúde expostos à doença coronavírus 2019. }\end{array}$ & 2020 \\
\hline Distúrbios do sono em profissionais da saúde no surto de covid - 19: & 2021 \\
\hline uma revisão integrativa da literatura. & \\
\hline
\end{tabular}

Fonte: Própria (2021)

\section{RESULTADOS E DISCUSSÃO}

O artigo Impact of COVID-19 on eating habits, physical activity and sleep in Brazilian healthcare professionals (MOTA, I. A., et al. 2021), mostrou que, os médicos estão mais insatisfeitos com a interferência dos problemas de sono nas suas atividades da vida diária do que outros profissionais de saúde. Em contraste, os profissionais não médicos relataram uma maior insatisfação com a percepção dos outros sobre a interferência do sono na sua qualidade de vida e estavam mais estressados com os seus problemas de sono.

Um outro estudo Impact of COVID-19 pandemic on the sleep quality of medical professionals in Brazil (BRITO-MARQUES, J. M. A. M. et al., 2021) identificou que a maioria dos médicos $(65,6 \%)$ apresentou alterações no sono, sendo a má qualidade do sono reportada por $73,1 \%$. Sintomas depressivos estiveram presentes em $75,8 \%$, e ansiedade em $73,4 \%$.

Ventura, (2021) mostrou no seu estudo "Distúrbios do sono em profissionais da saúde no surto de covid - 19: uma revisão integrativa da literatura." que a representatividade feminina foi de $61 \%$ e masculina $39 \%$ do total de participantes. A idade média da amostra foi de $28-40$ anos, sendo 35 anos a idade de destaque. Os enfermeiros foram os profissionais da saúde mais afetados pela insônia, sendo citados em quatro trabalhos (57\%). Inúmeros fatores podem contribuir para a qualidade do sono dos profissionais da saúde, como o desgaste físico pelo isolamento social, o uso de equipamento de proteção individual por muitas horas, preocupações em relação à família e o medo de contaminação.

O estudo de Phillippart et al. (2021) "Sleep disorders in healthcare professionals in the covid outbreak - 19: an integrative literature review" apontou que os enfermeiros foram os 
profissionais mais afetados pela insônia 57\%. E muitos fatores que contribuem para a qualidade do sono desses profissionais de saúde, são o desgaste físico pelo isolamento social, uso contínuo de Equipamento de Proteção Individual (EPIs), preocupações com relação à família e o medo de contaminação.

Ferreira et al (2021) mostraram o resultado de uma pesquisa, 24 pessoas, com idade entre 51 e 89 anos, de ambos os sexos. Foi possível verificar que a pandemia afetou o dia-dia das pessoas, apresentando tristeza $34,4 \%$, crises de ansiedade $25 \%$, estresse $13 \%$, medo de contrair a Covid-19 25\%, insônia 25\%, irritabilidade (26,1\%), aumento de apetite $16 \%$, além, de falar sozinho frequentemente $54 \%$.

O estudo "Insomnia and sleep quality in healthcare workers fighting against COVID19: a systematic review of the literature and meta-analysis", realizado por Serrano-Ripoll, M.J., et al (2021), foram identificados 18 estudos relevantes, após uma pesquisa bibliográfica em MEDLINE, EMBASE, e PsycINFO A prevalência de insónia estimada por meta-análise de efeitos aleatórios foi de 38\% (95\% CI= 37 a 39\%), sendo ligeiramente mais elevada nas mulheres $(29 \%, 95 \% \mathrm{CI}=27 \%$ a $30 \%)$ do que nos homens $(24 \%, 95 \% \mathrm{CI}=21$ a $27 \%)$. O principal fato de risco associado à insônia era trabalhar num ambiente de alto risco, seguido pelo sexo feminino e ter um nível educacional mais baixo.

Sanghera et al. (2020) The impact of SARS-CoV-2 on the mental health of healthcare workers in a hospital setting-A Systematic Review" na sua revisão sistemática, constou com a inclusão de 44 estudos, sendo revisões sistemáticas e metanálises, investigou a prevalência da condição de saúde mental dos trabalhadores da saúde e o impacto dos resultados nesses profissionais durante a pandemia. O objetivo desse trabalho foi identificar o impacto e os fatores de risco a fim de auxiliar em intervenções futuras. Os autores observaram a prevalência de condições de saúde mental nos trabalhadores de saúde durante a pandemia em 15 países. Essas condições eram depressão, ansiedade, insônia e reação aguda ao estresse.

Foi encontrado no estudo de Jahrami, et al. (2021) "O exame da qualidade do sono para os profissionais de saúde da linha de frente durante o surto de COVID-19" um total de 257 participantes (129 participantes da linha de frente da saúde e 128 trabalhadores de saúde não linha de frente), os trabalhadores da linha de frente da saúde apresentavam $85 \%$ estresse moderado-grave e $61 \%$ apresentavam má qualidade do sono e estresse moderado-grave. Para os trabalhadores de saúde não linha de frente, $84 \%$ tinham estresse moderado-grave e $62 \%$ tinham má qualidade do sono e estresse moderado-grave. Sexo feminino e formação profissional foram os preditores da má qualidade do sono e estresse. 


\section{A QUALIDADE DO SONO DOS PROFISSIONAIS DE SAÚDE}

No estudo de Xiao, et al. (2020) "The effects of social support on sleep quality of medical staff treating patients with coronavirus disease 2019 (COVID-19) in January and February 2020 in China." são encontradas alterações na rotina de sono como um importante fator que impacta a saúde mental. Os resultados mostraram também que os níveis de apoio social referidos pelos participantes eram associados ao sentimento de autoeficácia no trabalho e à qualidade do sono. $\mathrm{O}$ estudo apontou que os níveis de ansiedade se associavam aos níveis de estresse, impactando negativamente o senso de autoeficácia e a qualidade do sono dos profissionais. É percebido que atuar na linha de frente do combate à pandemia do novo corona vírus trouxe à tona aumentos dos níveis de ansiedade e estresse, como ao declínio da autoeficácia, e que essas variáveis influenciavam negativamente a qualidade do sono e do apoio social percebido pelos profissionais de saúde que atuavam na linha de frente.

Outro estudo realizado por Pappa, et al. (2020). Foram analisados meta-análises de 13 estudos transversais, com total de 33.062 participantes, fornecendo a evidência de que um grande número de profissionais de saúde experimentaram níveis significativos de ansiedade, depressão e insônia durante a pandemia. A ansiedade foi avaliada em 12 estudos, com uma prevalência combinada de $23,2 \%$, os dados de gênero estavam disponíveis em seis estudos, com uma prevalência de 20,92\% para homens e 29,06\% para mulheres. Com relação à gravidade da ansiedade, os dados estavam disponíveis em seis estudos com uma prevalência combinada de $17,93 \%$ para ansiedade leve e $6,88 \%$ para moderada/grave.

LAI, J. et al. (2020) realizou um estudo sobre "Fatores associados aos resultados de saúde mental entre os profissionais de saúde expostos à doença coronavírus 2019” com 1.257 profissionais de saúde em 34 hospitais equipados com clínicas ou enfermarias para pacientes com COVID-19 em várias regiões da China, e foi encontrado uma proporção considerável de profissionais de saúde com sintomas de depressão, ansiedade, insônia e angústia. Entre o grupo que mais sofria estavam as mulheres, enfermeiras, pessoas que moravam em Wuhan e profissionais de saúde envolvidos no diagnóstico, no tratamento ou na prestação de cuidados de enfermagem a pacientes com suspeita ou confirmação de COVID-19.

Figura 01: Descrição das complicações que a insônia pode causar. 
LIMA, et al.

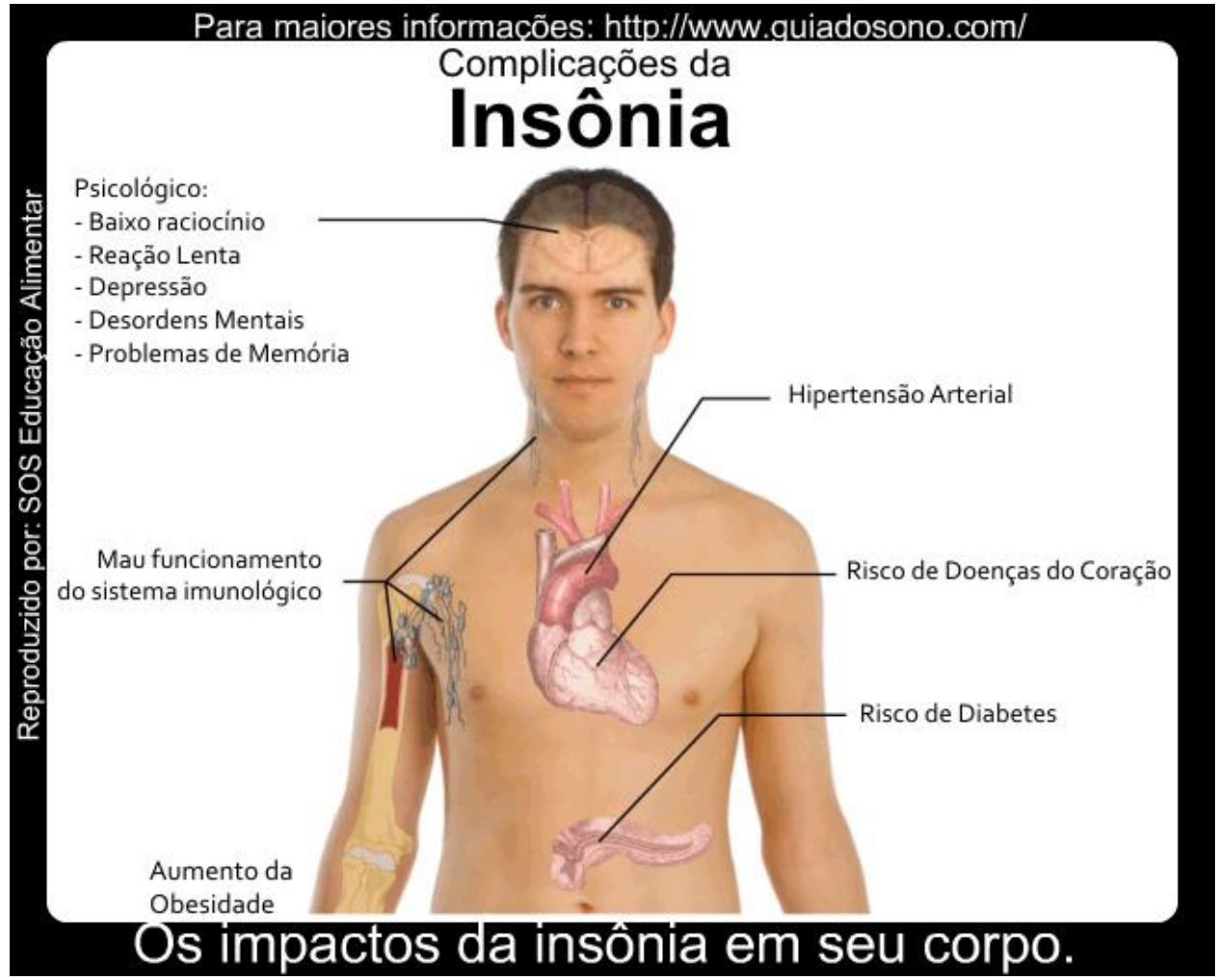

Fonte: Google

A figura 01 mostra de forma clara, os impactos causados pela complicação da insônia. As preocupações excessivas com os problemas do dia a dia podem deixar seu cérebro ativo durante a noite, levando em consideração o fato de estarmos vivenciando uma pandemia avassaladora, acaba sendo comum as preocupações surgirem, o que acaba dificultando o sono e causa a insônia, outros efeitos da insônia em longo prazo são irritação, dificuldade para se concentrar ou de memória, sintomas de depressão, entre outras.

A insônia pode gerar um impacto negativo no desempenho do trabalho dos profissionais da saúde, além da dependência de tratamentos farmacológicos, a longo prazo. De acordo com a pesquisa publicado pelo Jornal da USP (2021), sem mudanças nas condições às quais esses trabalhadores são expostos, erros de ordem médica e de atenção no cuidado aos pacientes, além da possibilidade do desenvolvimento e agravo de problemas de saúde, podem ser esperados.

Com os profissionais de saúde não foram diferentes, o fato de estar na linha de frente, a preocupação de se infectar ou de ser o transmissor do vírus para a sua casa, com certeza é aterrorizante, mas a questão é que era difícil imaginar que a pandemia fosse se estender tanto, principalmente aqui no país. A falta de políticas governamentais eficientes somada ao atraso da vacinação resultaram em um recorde diário de mortes que traz o medo frequente de ser infectado e precisar de cuidados intensivos.

Durante o sono são produzidas citocinas, proteínas responsáveis pelas defesas do organismo contra os processos infecciosos como os da COVID-19. Nesse período de descanso, 


\section{A QUALIDADE DO SONO DOS PROFISSIONAIS DE SAÚDE}

ainda são restauradas as funções cognitivas do cérebro que permitem concentração nas tarefas do dia a dia e trazem bem-estar, diminuindo o risco de quadros de depressão. O estado emocional tem um papel importante na qualidade do sono, então, o sono é o momento de recuperação do organismo, com a eliminação de alguns hormônios que permitem a regeneração dos grupos musculares. E no sono profundo descansamos nossa mente e recuperamos nosso estado emocional (SAÚDE ATIVA, 2020).

Não dormir bem, o estresse e os problemas de saúde mental entre os profissionais de saúde podem prejudicar suas habilidades cognitivas e suas tomadas de decisões clínicas, aumentando os riscos de cometerem erros clínicos que possam aumentar os riscos para os pacientes, cuidar da saúde mental dos profissionais de saúde durante a pandemia deve ser uma preocupação crucial para a saúde pública. O desenvolvimento e a implementação da avaliação, apoio e intervenção em saúde mental tornam-se essenciais para gerenciar as consequências para a saúde mental durante o surto e depois.

\section{CONCLUSÕES}

Os estudos mostraram que os profissionais de saúde foram afetados no seu dia a dia, tristeza, crises de ansiedade, e o comparativo entre homens e mulheres, as mulheres tiveram um número ligeiramente elevado mais do que os homens.

Dormir bem é uma condição essencial para contribuição da qualidade de vida de qualquer pessoa, é notório o impacto que a pandemia causou com relação a privação do sono e quando essa privação do sono afeta os profissionais de saúde, os danos podem ser mais graves, pois estes são responsáveis por outras vidas, que estão sob a sua responsabilidade.

Além disso, todos precisam atentar-se para a real importância do sono para a saúde humana, entre sono e indicadores de saúde, o mais importante que a duração do sono é a qualidade sono.

\section{REFERÊNCIAS}

BARROS, M.B. A. et al. Relato de tristeza/depressão, nervosismo/ansiedade e problemas de sono na população adulta brasileira durante a pandemia de COVID-19. Epidemiologia e Serviços de Saúde [online]. v. 29, n. 4. Disponível em: <https://doi.org/10.1590/S1679$49742020000400018>$. Acesso em 14 de Junho de 2021.

BLUME,C., SCHMIDT, M.H., e CAJOCHEN C. Effects of the COVID-19 lockdown on human sleep and rest-activity rhythms. Current Biology, 2020, 30(14), 795-797. 
LIMA, et al.

BRITO-MARQUES, J. M. A. M. et al. Impact of COVID-19 pandemic on the sleep quality of medical professionals in Brazil. Arquivos de Neuro-Psiquiatria [online]. 2021, v. 79, n. 2, pp. 149-155. Available from: <https://doi.org/10.1590/0004-282X-anp-2020-0449>. Epub 19 Mar 2021. ISSN 1678-4227. https://doi.org/10.1590/0004-282X-anp-2020-0449. Acesso em 03 de agosto de 2021.

CESPEDES, M.S., e SOUZA, J.C.R.P. Hábitos e distúrbios do sono nos estudantes de medicina da Universidade Estadual de Mato Grosso do Sul. Arquivos Médicos dos Hospitais e da Faculdade de Ciências Médicas da Santa Casa de São Paulo,2020, 66(2), 116-123.

COMO SUPERAR A INSÔNIA E DORMIR BEM, MESMO DURANTE A PANDEMIA. SulAmérica Saúde Ativa,2020. Acesso em 01 de Setembro de 2021.

DIAS, E. A Educação, a pandemia e a sociedade do cansaço. Ensaio: Avaliação e Políticas Públicas em Educação [online]. 2021, v. 29, n. 112 , pp. 565-573. Disponível em: <https://doi.org/10.1590/S0104-40362021002901120001>. Epub 05 Jul 2021. ISSN 18094465. https://doi.org/10.1590/S0104-40362021002901120001. Acesso em 16 Julho 2021.

FERREIRA, A. S. et al, Impacto da pandemia da covid-19 na qualidade de vida dos idosos, Portal de Periódicos São Lucas, 2021 ,Rôndonia, Porto Velho. Disponível em < Open Journal Systems (saolucas.edu.br)>. Acesso em 01 de Agosto de 2021.

JAHRAMI, H., et al. O exame da qualidade do sono para os profissionais de saúde da linha de frente durante o surto de COVID-19. Sono \& respiração = Schlaf \& Atmung vol. 25,1 (2021): 503-511. doi:10.1007/s11325-020-02135-9. Disponível em < $\underline{\text { O exame da qualidade do sono }}$ para os profissionais de saúde da linha de frente durante o surto de COVID-19 - PubMed (nih.gov)>. Acesso em 20 de agosto de 2021.

KANG, L. et al. A saúde mental dos médicos em Wuhan, China, lidando com o novo coronavírus de 2019. A lanceta. Psiquiatria vol. 7,3 (2020): e14. doi:10.1016/S22150366(20)30047-X. Disponível em < A saúde mental dos médicos em Wuhan, China, lidando com o novo coronavírus 2019 - PubMed (nih.gov)> Acesso em 20 de Agosto de 2021. 


\section{A QUALIDADE DO SONO DOS PROFISSIONAIS DE SAÚDE}

LAI, J. et al. Fatores associados aos resultados de saúde mental entre os profissionais de saúde expostos à doença coronavírus 2019. Rede JAMA abre vol. 3,3 e203976. 2 mar. 2020, doi:10.1001/jamanetworkopen.2020.3976. Disponível em < Factors Associated With Mental Health Outcomes Among Health Care Workers Exposed to Coronavirus Disease 2019 PubMed (nih.gov)>. Acesso em 20 de agosto de 2021.

LIMA, M. G. et al. Associação das condições sociais e econômicas com a incidência dos problemas com o sono durante a pandemia de COVID-19. Cadernos de Saúde Pública [online]. 2021, v. 37, n. 3 Acesso em 15 de Junho 2021.

MOTA, I. A., et al. Impact of COVID-19 on eating habits, physical activity and sleep in Brazilian healthcare professionals. Arquivos de Neuro-Psiquiatria [online]. 2021, v. 79, n. 5 [Accessed 3 August 2021] , pp. 429-436. Available from: <https://doi.org/10.1590/0004-282XANP-2020-0482>. Epub 24 May 2021. ISSN 1678-4227. https://doi.org/10.1590/0004-282XANP-2020-0482.

ORBEN, A., TOMOVA, L., e BLAKEMORE, S. J. (2020). The effects of social deprivation on adolescent developmentand mental health. The Lancet, 4(8),634-640.

PAPPA, S. et al. Prevalência de depressão, ansiedade e insônia entre os profissionais de saúde durante a pandemia COVID-19: Revisão sistemática e meta-análise. Cérebro, comportamento e imunidade vol. 88 (2020): 901-907. doi:10.1016/j.bbi.2020.05.026. Disponível em <Prevalência de depressão, ansiedade e insônia entre os profissionais de saúde durante a pandemia COVID-19: Revisão sistemática e meta-análise - PubMed (nih.gov)>. Acesso em 20 de Agosto de 2021.

PHILIPPART, K.S., et al., Sleep disorders in healthcare professionals in the covid outbreak 19: : an integrative literature review. Health and Society, v. 1, n. 03, Jul. 2021. Disponível em $<$ SLEEP DISORDERS IN HEALTHCARE PROFESSIONALS IN THE COVID OUTBREAK - 19: | Health and Society (periodicojs.com.br)>. Acesso em 20 de Agosto de 2021.

PRADO, A. D., PEIXOTO, B.C., DA SILVA, A. M. B., SCALIAL, A.M. A saúde mental dos profissionais de saúde frente à pandemia do COVID-19: uma revisão integrativa. Revista Eletrônica Acervo Saúde, n.46, 2020. Disponível em < A saúde mental dos profissionais de 
LIMA, et al.

saúde frente à pandemia do COVID-19:uma revisão integrativa. Revista Eletrônica Acervo Saúde(acervomais.com.br)>. Acesso em 20 de agosto de 2021.

RUIZ, F.S.A e TUFIK, S. Aspectos imunológicos do sono. In: Paiva T, Andersen ML, Tufi k S, eds. O sono e a medicina do sono. Barueri: Manole; 2014. p.124-3.

SANGHERA, J.et al., The impact of SARS-CoV-2 on the mental health of healthcare workers in a hospital setting-A Systematic Review. J Occup Health, v. 62, n 1, 2020. Disponível em $<\underline{\text { O impacto do SARS-CoV-2 na saúde mental dos profissionais de saúde em uma revisão }}$ sistemática hospitalar. | J Occup Health;62(1): e12175, 2020 Jan. | MEDLINE (bvsalud.org)>. Acesso em 20 de Agosto de 2021.

SERRANO-RIPOLL, M.J. et al (2021) Insomnia and sleep quality in healthcare workers fighting against COVID-19: a systematic review of the literature and meta-analysis, Actas espanolas de psiquiatria. Disponível em < Insomnia and sleep quality in healthcare workers fighting against COVID-19: a systematic review of the literature and meta-analysis. (epistemonikos.org)>. Acesso em 04 de Agosto de 2021.

VENTURA, T. L. et al. 2021. Distúrbios do sono em profissionais da saúde no surto de covid - 19: uma revisão integrativa da literatura. Estudos Avançados Sobre Saúde E Natureza, 1. Disponível em < https://periodicojs.com.br/index.php/easn/article/view/189. $>$ Acesso em $20 \mathrm{de}$ Agosto de 2021.

WORLD HEALTH ORGANIZATION. WHO Coronavirus Disease (COVID-2019) situation reports. Disponível em: https://www.who.int/emergencies/diseases/novelcoronavirus-2019/situation-reports

» https://www.who.int/emergencies/diseases/novel-coronavirus-2019/situation-reports. Acesso em 16 de Junho de 2021

XIAO, H. et. al., 2020 . The effects of social support on sleep quality of medical staff treating patients with coronavirus disease 2019 (COVID-19) in January and February 2020 in China. Medical Science Monitor, 26, e923549. http://dx.doi.org/10.12659/MSM.923549. Disponível em < Os Efeitos do Apoio Social na Qualidade do Sono da Equipe Médica Que Trata pacientes com Doença Coronavírus 2019 (COVID-19) em janeiro e fevereiro de 2020 na China - PubMed 


\section{A QUALIDADE DO SONO DOS PROFISSIONAIS DE SAÚDE}

(nih.gov)>. Acesso em 20 de Agosto de 2021. 